\title{
Mapping gastric dysrhythmias in gastroparesis-a slow wave of electrical activity
}

S cientists have accurately tracked the patterns of abnormal gastric electrical activity that occur during gastroparesis using high-resolution novel technology adapted from cardiac mapping. The new findings, published in Gastroenterology, offer a detailed description of the abnormalities that occur during gastric dysrhythmias, and provide a novel classification system for these abnormal patterns.

"We provided the first accurate, high-resolution description of gastric dysrhythmias in humans," notes first author Gregory O'Grady from the University of Auckland, New Zealand. "A range of abnormal slow-wave initiation and conduction patterns were revealed, in both diabetic and idiopathic gastroparesis," he adds.

Abnormal gastric electrical activity is already known to be associated with gastroparesis, but no accurate descriptions of these abnormalities exist and previous work has been hampered by the lack of adequate methods to investigate gastric electrical activity.

"We decided to take a fresh look at electrical dysrhythmia in gastroparesis, using novel technology adapted from cardiac mapping," explains O'Grady. Taking inspiration from cardiac electrophysiology, the researchers adopted a multidisciplinary approach and developed electrodes for highresolution gastric mapping and new analysis algorithms for interpretation of the results.

Created from flexible print circuit boards, each array contained 256 electrodes spaced $4 \mathrm{~mm}$ from each other, enabling the researchers to track patterns of gastric electrical activity in fine spatiotemporal resolution. "These arrays must be placed directly on the serosa of the stomach, which was possible in this study because the participants were undergoing gastric electrical stimulator implantation," says O’Grady.

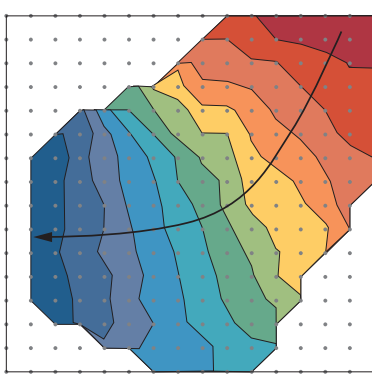

Normal antegrade propagation

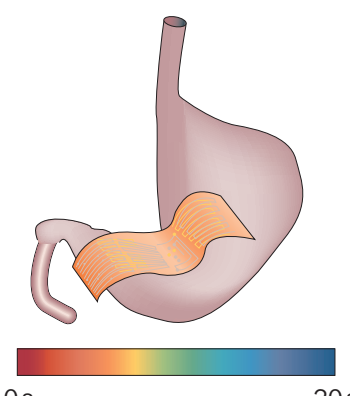

Os

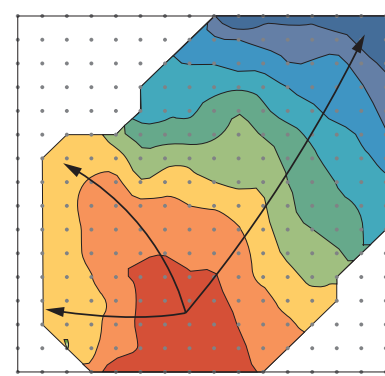

$20 \mathrm{~s}$ Ectopic activation with tachygastria

High-resolution electrical mapping of gastric electrical activity. Courtesy of G. O'Grady.

First, the researchers recruited 12 patients with either diabetic $(n=8)$ or idiopathic $(n=4)$ gastroparesis to their study who were due to undergo gastric electrical stimulation. Prior to stimulator implantation, electrode arrays were placed on the serosa of the stomach and gastric electrical activity recorded (for an average $13.4 \mathrm{~min}$ ). The collected data were then analysed using tailored electrical mapping software and compared with reference control data obtained previously. Gastric biopsy samples were also taken so that the number of interstitial cells of Cajal (ICC) could be quantified.

O'Grady et al. found that slow-wave abnormalities were detected in nearly all of the patients tested (11 of 12), ranging from minor transient deviations to highly disorganized and persistent patterns. Moreover, these patterns could be categorized into either abnormal initiation (diffuse focal events or stable ectopic pacemaking) or abnormal conduction (reduced velocities or conduction blocks), and then further subclassified according to pattern, rhythm and rate of activity.

Interestingly, both of the above categories often co-existed and a large proportion of patients (7 of 12) had normal slow-wave frequencies throughout their recordings, which did not dictate normal propagation patterns. No difference was observed in dysrhythmia patterns between the two types of gastroparesis studied. Furthermore, mean ICC counts were markedly reduced in patients with gastroparesis compared with matched individuals as controls.

"We found that human gastric electrical abnormalities often occurred at a normal slow-wave frequency, meaning that they could be missed by other methods lacking spatial resolution, such as electrogastrography," notes O'Grady, who believes the abnormal propagation patterns they observed might be explained by loss of ICCs.

O'Grady notes that the new findings are a lasting testament to the hard work of all the investigators involved, but especially co-author Andrew Pullan who sadly died this year: "With great sadness, one of the principal investigators and visionaries for the work, Professor Andrew Pullan, lost his battle with cancer during the final stages of the study."

The authors now hope that their study will be the first of many to use this new technology. They also hope that the technical advances described will contribute to an improved understanding of the underlying pathophysiology of gastroparesis, elucidation of which has thus far been challenging.

\section{Katrina Ray}

